\title{
Thermal fatigue analysis of automotive Diesel piston: Experimental procedure and numerical protocol
}

\author{
F. Szmytka ${ }^{\mathrm{a}, *}$, M. Salem ${ }^{\mathrm{b}}$, F. Rézaï-Aria ${ }^{\mathrm{b}}$, A. Oudin ${ }^{\mathrm{a}}$ \\ a PSA Peugeot Citroën, Centre Technique de Vélizy, route de Gisy, 78943 Vélizy Villacoublay, France \\ ${ }^{\mathrm{b}}$ Université de Toulouse, Mines Albi, INSA, UPS, ISAE, ICA (Institut Clément Ader), Campus Jarlard, F-81013 Albi, France
}

\begin{abstract}
A B S T R A C T
A new thermal fatigue rig using High Frequency induction heating is developed to test automotive Diesel engine pistons. An adapted test piston is internally cooled by permanent water flow while its bowl sensitive to thermo-mechanical fatigue is subjected to cyclic induction heating. The temperature is measured in depth by thermocouples and in surface by a thermal infrared camera or a pyrometer. The crack initiation and propagation and the local deformations are provided by optical means. Thermo-mechanical loadings are calibrated by thermal measurements on the piston during engine operation and the entire test is modelled by finite elements. A constitutive model and a fatigue criterion for aluminium alloys are proposed to estimate the piston lifetime under severe cyclic loading. The proposed fatigue bench allows loading the piston in thermal fatigue scheme very similar to that encountered in engine operation conditions. Crack detection is facilitated by numerical modelling that helps to detect the most critical areas and also to reliably estimate the number of cycles for initiate cracks.
\end{abstract}

Keywords:

Thermal fatigue

Thermal fatigue rig

Automotive pistons

Aluminium alloys

Fatigue criterion

\section{Introduction}

The design of components to increase their Thermal-Mechanical Fatigue (TMF) resistance is nowadays a major scientific issue in an industrial point of view. Reliability has become a crucial point particularly in the automotive industry as it is now necessary to offer the most reliable components to customers in a very competitive market. In the same time, for performance and ecological considerations car engines tend to be exposed to higher temperatures and pressures during their nominal use. Taking into account of these evolutions, the choice of a design and of an associated material for an engine part has to be accurate in the early conception stages and engineers should have access to an efficient experimental basis to quickly assess alternative geometries, materials or processes.

Under normal operating conditions, automotive pistons undergo severe thermal, mechanical and physical-chemical loadings as playing a central part in the combustion process. Fatigue damage occurs in different regimes - high cycle and low cycle one- and is often the result of a complex coupling between temperature, thermal gradients, mechanical loading conditions, combustion pressure and even chemical combustion reactions [1-7]. Only Thermal Fatigue (TF) or "Thermal Stresses" is considered here as temperature cycling induces transient thermomechanical

\footnotetext{
* Corresponding author.

E-mail address: fabien.szmytka@mpsa.com (F. Szmytka).
}

strains and stresses particularly on some critical points of the structure.

The automotive piston is indeed a highly thermomechanically loaded part that requires specific geometry for Diesel engines in order to improve performances and to reduce polluting emissions by optimising the combustion chemical reactions [8,9]. From a thermal cycle point of view, a piston is first heated, during the engine operation, by forced convection with the combustion flux and then cooled by forced convection with engine oil and conduction with the rings and the cylinder block. These heat exchanges generate transient loading cycles in the component, which, coupled with the severe external mechanical loads, induce transient thermal-mechanical loadings. The repetition of these transients thermomechanical cycling is responsible for the piston cracking $[3-7,10,11]$. On this topic, a new TF set-up is developed to simulate experimentally these transient thermal loads on laboratory piston.

For designing automotive engines part, prototypes are usually used on test bench for applying load in complex but close to real conditions $[5,11]$. These test benches are usually not very flexible in terms of loading conditions, difficult to set up and expensive. Very few studies mention other TF experimental set-ups. Song et al. [12,13] developed a special test bench using a laser heating, a method that was also used for hot-work tooling [14]. Lasers allow producing very local heating and thermal gradients are generated by the bulk part (thermal inertia). This work [12,13] only deals with the study of the thermal and thermo-mechanical loadings 
but damage analysis is partial and could need enhancement. An experimental setup to observe TF damage on pistons using induction heating could also be found in [15] but without detailed description. Heating is applied, in this case, at the bowl edge while the bottom of the piston is cooled by immersion in a water bath. The piston lifetime is then estimated by the number of cycles necessary for the growth of a $1.5 \mathrm{~mm}$-long crack.

All these propositions do not easily allow making the link between the laboratory piston test and the real load observed in nominal conditions. The TF test bench developed here is able to test pistons with different geometries and materials reproducing as closely as possible the thermal loading in engine. The aim of the tests is to estimate the number of heating/cooling cycles required for the initiation and propagation of TF cracks. Finite element calculations are used to estimate the maximum temperatures observed during engine operation and to identify the thermal gradients in critical zones. The experimental thermal solicitation is then monitored to achieve the level of temperatures and to operate with gradients at least equal in severity. To simulate a representative thermal field, the piston is cooled by a permanent water flow through its cooling channel while the bowl is alternatively heated by induction. The test is thus developed so as to compare piston geometries or materials choice for a given loading.

In terms of lifetime, Isothermal Fatigue (IF) is considered as a special case of TF. It is believed that experiences under IF at the maximum temperature of a TF thermal cycle can reproduce the damage (crack initiation and propagation) and life under TF conditions. The well-known IF laws and criteria have therefore been applied to describe TF crack initiation life. However, such approach requires to correctly describe the relevant mechanical parameters that can only be calculated in TF based on a good knowledge of the temperature fields and the material constitutive laws. The computational numerical approach for the lifetime assessment of structures under thermomechanical loading adopted by Constantinescu et al. [16] and Szmytka et al. [17] is adapted to pistons. A finite element analysis undertaken in this paper aims at estimating the TMF lifetime of a piston specimen tested on the developed TF test bench. The calculations are performed under the assumption of a weak coupling between thermal and mechanical loadings. Thus, thermal calculation is first performed to best represent the thermal gradients and maximum temperatures achieved experimentally. The mechanical computations are then run by employing the calculated thermal fields as boundary conditions. The most critical element is then determined using a dissipated energybased criterion. In the present study, only crack initiation is addressed and numerical as well as experimental lifetime are compared and analysed.

\section{Thermal fatigue experiments}

\subsection{Thermal fatigue rig}

A new TF rig using induction heating is developed in order to compare piston geometries or materials selected for a given loading. Thermal test conditions are the closest to severe thermal conditions in engine operation. The facility presented in Fig. 1 includes a High Frequency (100-300 kHz) CELES $25 \mathrm{~kW}$ generator, an acquisition data system and a mobile frame for supporting the piston. Alternative heat flux is applied at the bowl edge (TF interest zones) by an internal solenoid coil. Pistons are cooled by natural air convection at their external surfaces and by cold water $\left(20^{\circ} \mathrm{C}\right)$ circulating continuously through their internal channels (cooling duct and holes) with a flow rate of $10 \mathrm{l} / \mathrm{min}$.

Fig. 1 shows pictures of a dummy piston mounted on the test rig with all control and measurement facilities for the TF test. The temperature measurements are performed through the section by thermocouples inserted into the piston and on the surface with an "AGEMA LW 880" infrared camera and bi-chromatic pyrometer. Crack initiation life and then growth rates are measured by means of a "Questar" long distance travelling microscope.

\subsection{Material and specimen}

TF tests are performed on pistons made with a hypereutectic aluminium-silicon alloy AlSi13Cu4 ( 120 HB), commonly used for the production of automotive Diesel piston; its chemical

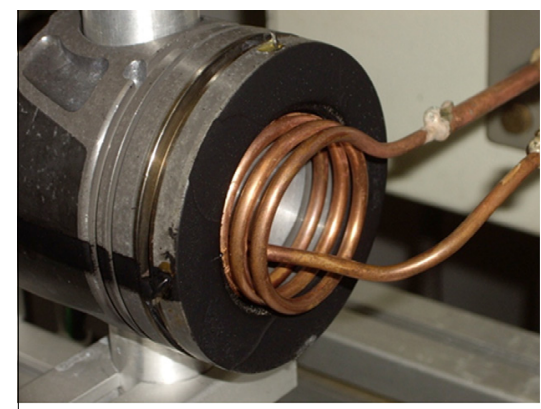

(a)

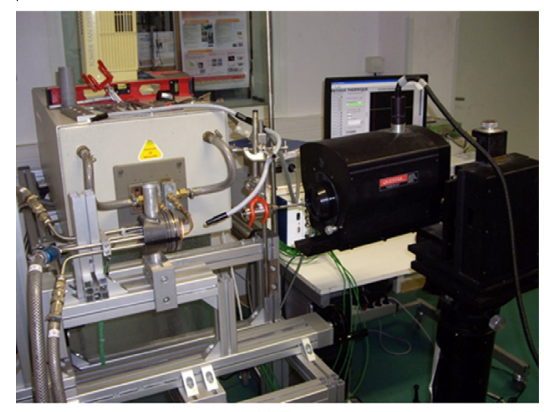

(c)

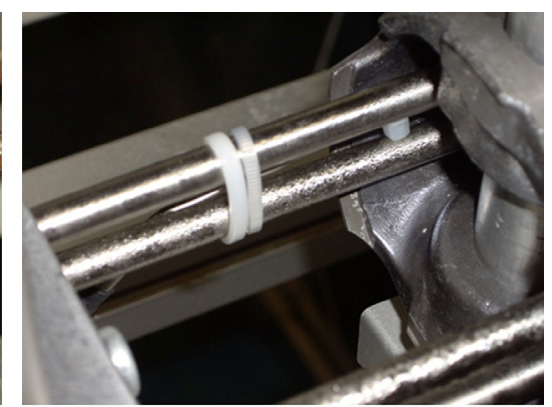

(b)

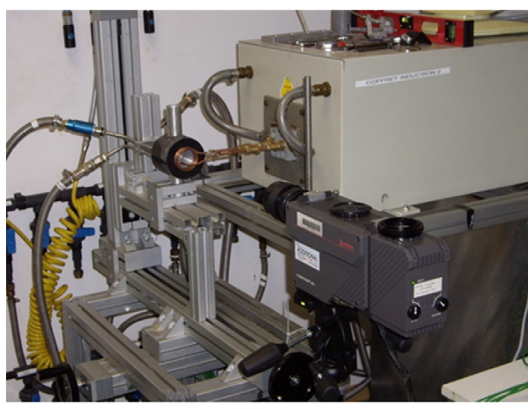

(d)

Fig. 1. Piston test bench. (a) Induction coil and bowl edge. (b) Cooling tubes. (c) General view with long distance microscope. (d) General view with infrared camera. 
Table 1

Chemical compositions of the used aluminium alloy (wt.\%).

\begin{tabular}{|c|c|c|c|c|c|c|c|c|c|c|}
\hline $\mathrm{Si}$ & Mn & $\mathrm{Fe}$ & $\mathrm{Mg}$ & $\mathrm{Cu}$ & $\mathrm{Zn}$ & $\mathrm{Ti}$ & $\mathrm{Ni}$ & Sn & $\mathrm{Cr}$ & $\mathrm{Zr}$ \\
\hline $12-14.5$ & $<0.20$ & $<0.70$ & $0.5-1.5$ & $3.7-5.2$ & $<0.10$ & $<0.20$ & $1.7-3.2$ & $<0.10$ & $<0.30$ & $<0.20$ \\
\hline
\end{tabular}

composition is given in Table 1 . The microstructure consists mainly of Al- $\alpha$ matrix (solid solution), angular and polygonal primary silicon particles and several intermetallic phases such as $\mathrm{Al}_{9} \mathrm{FeNi}$, $\mathrm{Al}_{3} \mathrm{NiCu}, \mathrm{Al}_{2} \mathrm{Cu}$ and $\mathrm{Al}_{5} \mathrm{Cu}_{2} \mathrm{Mg}_{8} \mathrm{Si}_{6}$.

This alloy provides a good compromise between its thermal and mechanical properties, which are all summed up in Table 2. We notice a significant drop in the mechanical properties above $300{ }^{\circ} \mathrm{C}$ with an ultimate tensile strength which only reaches $39 \mathrm{MPa}$ at $400^{\circ} \mathrm{C}$.

The TF test piston is represented in Fig. 2. Manufactured parts have undergone some modifications to be adapted to the new TF rig. The bottom of the bowl is milled so as to easily insert the solenoid coil for heating and thereby to prevent magnetic interference with the heating system. The piston was drilled at different locations to allow the insertion of thermocouples. These thermo sensors are designed to allow the centring of the heating system and the easy measurement of thermal gradients. A cooling system (stainless steel tubes) is fitted and glued into piston to the entrances of the cooling channel. It helps to reproduce, with increased severity, the cooling achieved by the oil in a Diesel engine combustion chamber.

Heating is done by the induction generator "CELES" generator by imposing a thermal spectrum that provides the desired evolution of temperature versus time in the thermocouple zones while the pyrometer enables to control the maximum temperature imposed on the bowl surface.

\subsection{Thermal fatigue test analysis procedure}

The piston thermal loading analysis is first performed using both a finite element simulation and thermal measurements (in engine use) to define a TF reference cycle ( $T_{\max }, T_{\min }$ and heating and cooling rates at prescribed locations). Then, the TF testing parameters are adjusted to reproduce those TF cycles on instru-

Table 2

Thermal and mechanical properties.

\begin{tabular}{lrrrr}
\hline & $20^{\circ} \mathrm{C}$ & $200{ }^{\circ} \mathrm{C}$ & $300{ }^{\circ} \mathrm{C}$ & $400{ }^{\circ} \mathrm{C}$ \\
\hline Young modulus (GPa) & 80 & 69 & 59 & 52 \\
Tensile Yield stress (at 0.2\% of strain) & 236 & 158 & 68 & 32 \\
$\quad(\mathrm{MPa})$ & & & & \\
Ultimate tensile strength (MPa) & 257 & 185 & 92 & 39 \\
Specific heat $\left(\mathrm{J} \mathrm{t}^{-1} \mathrm{~K}^{-1}\right)$ & 830 & 850 & 800 & 1000 \\
Conductivity $\left(\mathrm{W} \mathrm{m}^{-1} \mathrm{~K}\right)$ & 130 & 139 & 144 & 146 \\
\hline
\end{tabular}

mented piston unused as dummy specimen. The protocol consists in optimising the generator power supply cycle $P(t)$ to achieve the TF reference thermal cycle. TF tests are then carried out on pistons (with or without thermocouples) using optimised $P(t)$. TF tests are interrupted for observing the piston surface periodically in order to detect then follow the damage evolution (crack initiation and propagation). The temperature evolutions and crack growth are also monitored and recorded along the TF tests. After testing, pistons are cut for metallographic and fractographic analysis.

\section{FEM computational approach}

Different finite element simulations are performed for the preparation and analysis of the TF test. It is first necessary to simulate the thermal behaviour of a piston in case of standard operating conditions inside a Diesel engine. As mentioned previously, the simulation results are then used to adjust the experimental values as close as possible to the real heating and cooling rates. Thermal gradients are also measured to set the minimum level loading that will be imposed on the specimen. Finally, a thermo-mechanical simulation is performed to estimate, using the fatigue design tools (constitutive model and fatigue criterion) the number of cycles to failure as well as the most critical area of the piston specimen. This study allows validating the design protocol while identifying the zones to be observed for crack initiation detection.

\subsection{Thermal analysis}

\subsubsection{Engine thermal conditions}

The thermomechanical loading cycles applied to a piston usually correspond to an idle/full load cycle for the engine. The heating of the piston is usually related to the succession of combustion phases until a thermal equilibrium is achieved and maintained in the piston, which lasts about $10 \mathrm{~s}$. Then, as the engine is idled, the piston cools down naturally. There are consequently two computation stages in order to determine the stationary thermal map of the piston. First, a fluid flow full analysis of the engine combustion chamber including all the engine parts (piston, cylinder head, cylinder block and pipes) is undertaken. The four separate strokes - intake, compression, power, and exhaust - are computed to record the combustion heat flux applied to the piston. A post processing protocol enables to obtain a spatial map of the temporal mean heat transfer coefficients and combustion gas temperatures applied on the piston during a combustion cycle as seen in Fig. 3 . A Finite Element transient thermal analysis is then required to cor-
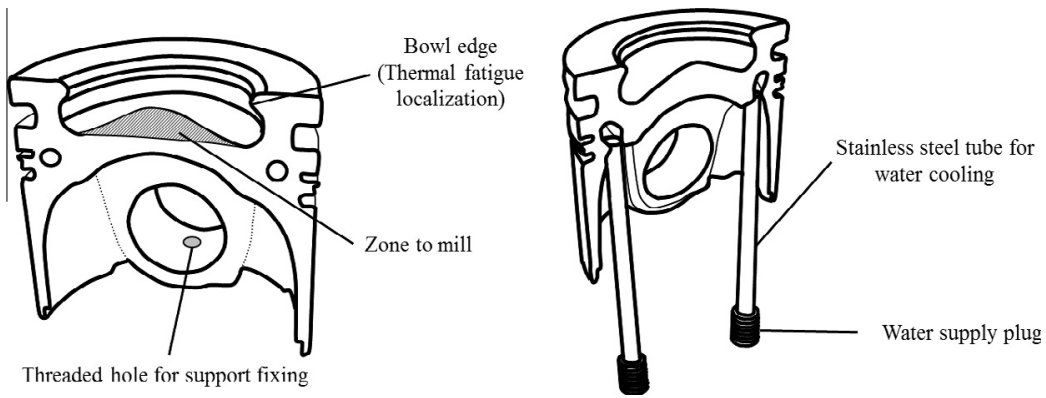

Fig. 2. Thermal fatigue test piston. 
(a)

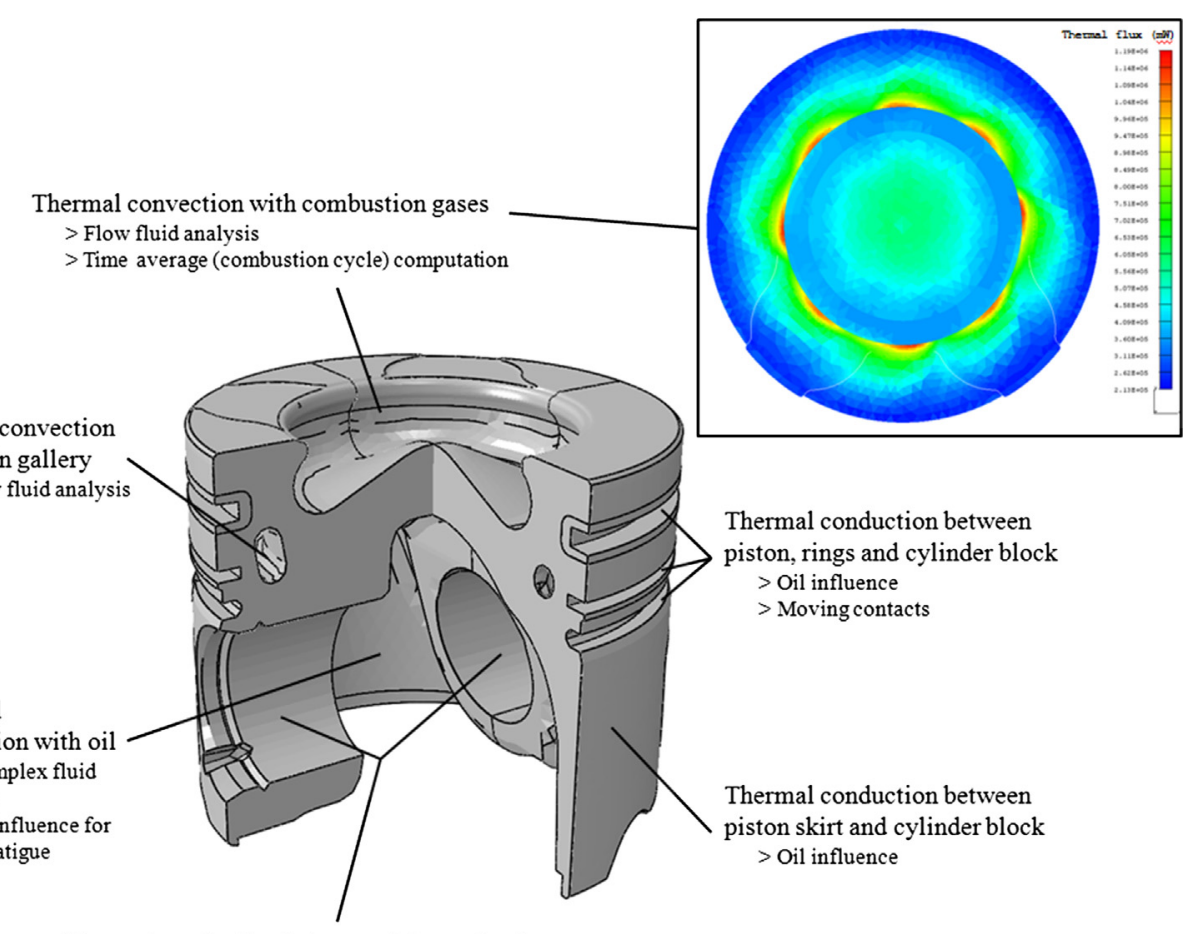

Thermal conduction between piston and axis $>$ Small influence for thermal fatigue

(b)

Surface heat flux

$>$ Optimisation to fit with experimental local temperature

$>$ Simple evaluation of the induction heating

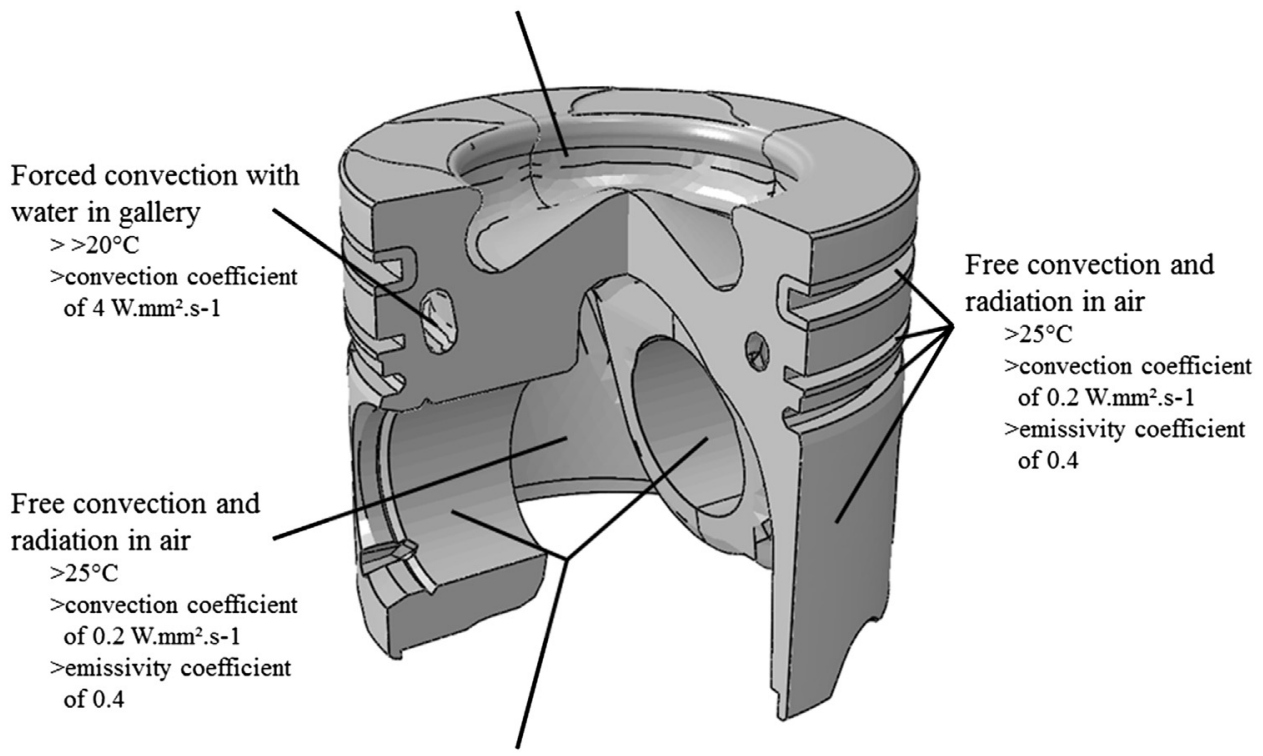

Adiabatic condition with the support

$>20^{\circ} \mathrm{C}$

Fig. 3. Thermal boundary conditions for a piston in a combustion chamber (a) and in the TF test bench (b).

rectly describe the piston heating-up and cooling-down cycles. The thermal boundary conditions are summarised in Fig. 3.

Five regions with an imposed convection and radiation were required to achieve a good correlation with thermal cycles measured in an engine. Sensitivity studies and experimental measurements have also helped to get a good representation of the convection transfers with the oil which extracts a significant part of the combustion heat flux. For this FEM simulation, the tetrahedral linear element meshing is created from a CAD drawing of the piston. The model is made of 273950 nodes and 164060 elements and consists in the piston, its rings and one fourth of the cylinder block.

\subsubsection{Bench thermal conditions}

Unlike engine conditions, the tested pistons are induction heated by electromagnetic field in particular in their "skin depth" with Foucault currents generating Joule effect. The induction frequency is about $100 \mathrm{kHz}$ and the estimated skin depth for the 
tested aluminium alloy is of about $0.2-0.4 \mathrm{~mm}$ for temperature ranging from 100 to $400{ }^{\circ} \mathrm{C}$. The thermal calculation does not exactly reproduce the real loading conditions that experience pistons. Indeed, to avoid a thermo-magnetic coupling computation, heating is modelled by a volume heat flux applied to the piston bowl edge. The applied flux intensity is optimised to obtain an appropriate matching between measured experimental and numerical thermal gradients. The heat exchanges with the water supply of the experimental setup are simulated here by imposing a forced convection in the cooling channel. The water temperature is set at $20^{\circ} \mathrm{C}$ and the convection coefficient used is $4 \mathrm{~mW} \mathrm{~mm}^{-2} \mathrm{~K}^{-1}$; it has been identified by a computational fluid dynamics analysis on a simplified gallery in steady-state condition. Free convection boundary conditions (exchange temperature of $25{ }^{\circ} \mathrm{C}$ for a convection coefficient of $0.02 \mathrm{~mW} \mathrm{~mm}^{-2} \mathrm{~K}^{-1}$ ) combined with radiation conditions $\left(25^{\circ} \mathrm{C}\right.$ for the piston environment and an emissivity coefficient of 0.4 ) are applied over the piston exterior surface in contact with air. Free convection coefficient for air is here set up to a common value used for this alloy and does not have a significant impact on the calculation. All these conditions are summed up on Fig. 3b.

\subsection{Thermomechanical analysis}

\subsubsection{Constitutive models}

The fields of thermal stress induced in the piston were calculated using a Thermo-Elasto-Visco-Plastic (EVP) constitutive model. EVP model was proposed by Szmytka et al. [17,18] to describe the behaviour of aluminium alloy examined over wide ranges of strain, strain rate and temperature. The basic assumptions for this model are small strains, isotropic elastic-visco-plastic behaviour and unified elastic-visco-plasticity thermodynamic framework. The constitutive model equations under isothermal conditions are reported in Table 3 while the evolution of $R_{0}$ with the temperature is detailed in Table 4 . Its values underline a very low elastic limit and thus a quick onset of viscoplasticity.

This model accurately reproduces strain-stress experimental hysteresis loops for different temperatures between ranging from $20{ }^{\circ} \mathrm{C}$ to $450{ }^{\circ} \mathrm{C}$ as shown in Fig. 4. The constitutive model parameters are identified by incremental cyclic strain/stress experiments [18]. These experiments enable to observe the inelastic cyclic behaviour of the alloy for different levels of strain amplitude. The identification basic cycle for a given level of strain $\varepsilon_{1}$ consists in a traction phase from 0 to $\varepsilon_{1}$, then the strain level $\varepsilon_{1}$ is maintained constant during $1800 \mathrm{~s}$ before returning back to 0 that once again remains constant for another $1800 \mathrm{~s}$. This basic cycle is repeated until the shakedown of the hysteresis loop is observed, which usually occurs after 5 cycles. Then, the strain level is increased and the cycling procedure is repeated again. With one specimen, 4 levels of strain (from $0.2 \%$ to $0.8 \%$ ) are explored at $20^{\circ} \mathrm{C}, 250{ }^{\circ} \mathrm{C}, 300^{\circ} \mathrm{C}$,
Table 4

Evolution of isotropic hardening parameter $R_{0}$ with temperature.

\begin{tabular}{lllll}
\hline & $20{ }^{\circ} \mathrm{C}$ & $200{ }^{\circ} \mathrm{C}$ & $300{ }^{\circ} \mathrm{C}$ & $400{ }^{\circ} \mathrm{C}$ \\
\hline$R_{0}(\mathrm{MPa})$ & 60 & 46 & 10 & 2 \\
\hline
\end{tabular}
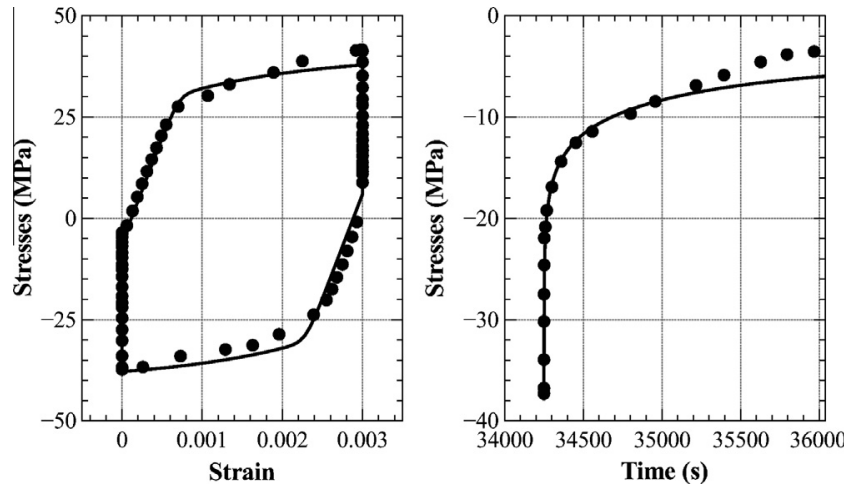

Fig. 4. Comparison between simulation and experimental results for cyclic hardening tests at $450^{\circ} \mathrm{C}$ (experimental data by dots while simulation is by solid line).

$350{ }^{\circ} \mathrm{C}$ and $400{ }^{\circ} \mathrm{C}$. Model viscous parameters are identified exclusively on cyclic loading with dwell phases.

\subsubsection{Fatigue damage criterion}

The experimental database which aims at observing the TMF behaviour of this alloy is constituted of 5 standard Low Cycle Fatigue (LCF) realised at $20^{\circ} \mathrm{C}$ and 14 Thermomechanical Fatigue uniaxial tests as described by Rémy [19]. For LCF experiments, the loading alternates traction and compression phases under strain controlled (with a strain ratio of $R_{\varepsilon}=-1$ ) conditions and at a constant mechanical strain rate. For TMF experiments, temperature and mechanical strain are simultaneously monitored for an outof-phase cycle as shown in Fig. 5 . Temperatures vary from $150{ }^{\circ} \mathrm{C}$ to $300{ }^{\circ} \mathrm{C}$ or from $200{ }^{\circ} \mathrm{C}$ to $380^{\circ} \mathrm{C}$ with dwell phases at maximum or maximum and minimum temperatures.

Experimental conditions are summed up in Table 5. For each test, the evolution of the maximum stress versus the number of cycles is recorded. It was observed that for all experiments the cyclic behaviour very quickly achieves an inelastic shakedown. Specimen is considered as damaged and achieved its lifetime when the stabilized maximum stress evolution vs. cycle presents a drop of $10 \%$. The lifetimes obtained in these experiments ranged between 3 and 2500 cycles.

A fatigue lifetime criterion used here is based on dissipated energy as proposed by previous authors [16-18,20-22]. Under LCF conditions, this approach was proven to have a physically

Table 3

Constitutive equations of the EVP model.

\begin{tabular}{|c|c|}
\hline Strain decomposition & $\underline{\underline{\varepsilon}}=\underline{\underline{\varepsilon}}^{e}+\underline{\underline{\varepsilon}}^{\text {ine }}+\underline{\underline{\varepsilon}}^{\text {th }}$ \\
\hline Yield function & $f(\underline{\underline{s}}-\underline{\underline{X}}, R)=J_{2}(\underline{\underline{s}}-\underline{\underline{X}})-R, \underline{\underline{s}}=\operatorname{dev}(\underline{\underline{\sigma}}) f(\underline{\underline{s}}-\underline{\underline{X}}, R)=J_{2}(\underline{\underline{s}}-\underline{\underline{X}})-R, \underline{\underline{s}}=\operatorname{dev}(\underline{\underline{\sigma}})$ with $\mathrm{J}_{2}$, the tensor second invariant \\
\hline Thermal strain & $\underline{\varepsilon}^{t h}=\alpha_{T}\left(T-T_{\text {ref }}\right) \underline{\underline{1}}$, with $\alpha_{T}$ the thermal expansion coefficient, $T$ the actual temperature et $T_{\text {ref }}$, a reference temperature \\
\hline Elastic strain & $\underline{\underline{\varepsilon}}^{e}=\underline{\underline{\underline{\underline{C}}}}^{-1}: \underline{\underline{\sigma}}$ \\
\hline Non linear kinematic hardening & $\underline{\underline{X}}=\frac{2 C}{3} \underline{\underline{\alpha}}, \underline{\underline{\alpha}}=\underline{\underline{\dot{\varepsilon}}}^{\text {ine }}-\frac{3 D}{2 C} \dot{p}$ with $C$ and $D$ temperature-dependent parameters \\
\hline Isotropic hardening & $\bar{R}=R_{0}+\bar{Q}(1-\exp (-\mathrm{bp}))$ with $R_{0}, Q$ and $b$ temperature-dependent parameters \\
\hline Flow rules & 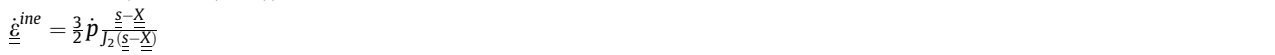 \\
\hline
\end{tabular}




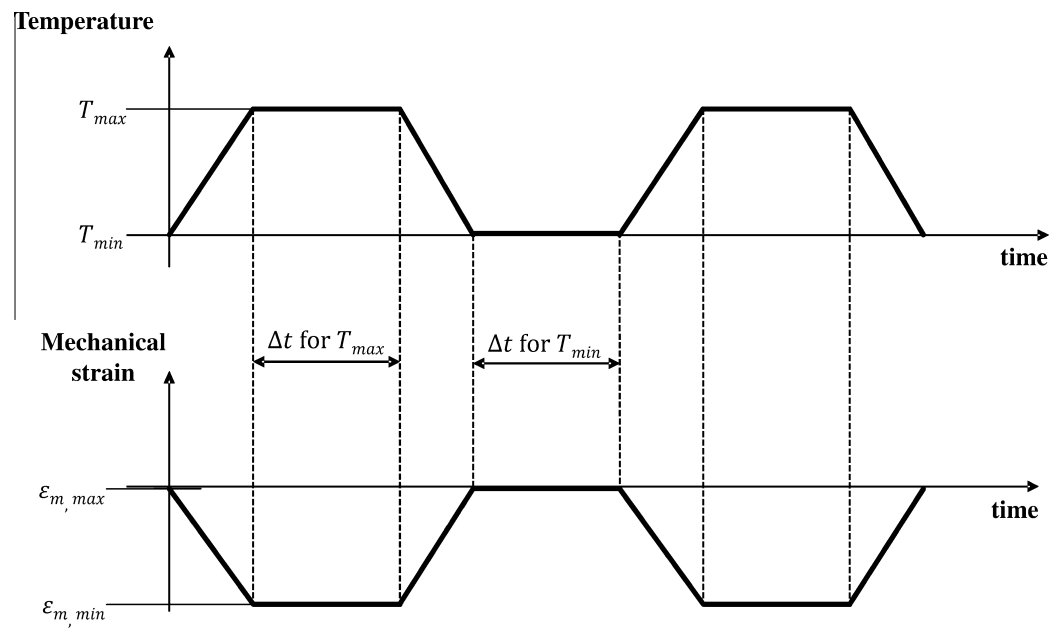

Fig. 5. Temperature and mechanical strain evolution for TMF tests.

Table 5

Experimental basis.

\begin{tabular}{|c|c|c|c|c|c|c|c|c|}
\hline \multicolumn{3}{|c|}{ Temperature $\left({ }^{\circ} \mathrm{C}\right)$} & \multicolumn{2}{|l|}{$\varepsilon_{\max }(\%)$} & \multicolumn{2}{|c|}{ Strain rate $\left(\mathrm{s}^{-1}\right)$} & $R_{\varepsilon}$ & \multirow[t]{2}{*}{ Number of tests } \\
\hline \multicolumn{8}{|l|}{ LCF tests } & \\
\hline \multirow[t]{3}{*}{20} & & & 0.25 & & \multirow[t]{3}{*}{$10^{-3}$} & & \multirow[t]{3}{*}{-1} & 2 \\
\hline & & & 0.5 & & & & & 2 \\
\hline & & & 1 & & & & & 1 \\
\hline$\varepsilon_{m, \max }(\%)$ & $\varepsilon_{m, \min }(\%)$ & $T_{\min }\left({ }^{\circ} \mathrm{C}\right)$ & $\Delta T, T_{\min }(\mathrm{s})$ & $T_{\max }\left({ }^{\circ} \mathrm{C}\right)$ & $\Delta T, T_{\max }(\mathrm{s})$ & Temperature rate $\left({ }^{\circ} \mathrm{Cs}^{-1}\right)$ & Mechanical strain rate & Number of tests \\
\hline \multicolumn{9}{|l|}{ TMF tests } \\
\hline 0 & -0.45 & 150 & 0 & 300 & 0 & 5 & $1.5 \cdot 10^{-4} \mathrm{~s}^{-1}$ & 2 \\
\hline 0 & -0.45 & 200 & 0 & 380 & 0 & 6 & $1.5 \cdot 10^{-4} \mathrm{~s}^{-1}$ & 2 \\
\hline 0 & -0.60 & 150 & 0 & 300 & 0 & 5 & $2.0 \cdot 10^{-4} \mathrm{~s}^{-1}$ & 2 \\
\hline 0 & -0.60 & 200 & 0 & 380 & 0 & 6 & $2.0 \cdot 10^{-4} \mathrm{~s}^{-1}$ & 2 \\
\hline 0 & -0.45 & 200 & 0 & 380 & 60 & 6 & $1.5 \cdot 10^{-4} \mathrm{~s}^{-1}$ & 2 \\
\hline 0 & -0.60 & 200 & 0 & 380 & 60 & 6 & $2.0 \cdot 10^{-4} \mathrm{~s}^{-1}$ & 2 \\
\hline 0 & -0.45 & 200 & 60 & 380 & 60 & 6 & $1.5 \cdot 10^{-4} \mathrm{~s}^{-1}$ & 2 \\
\hline
\end{tabular}

bases sound when the life is mainly controlled by micro-crack growth [19,21-27]. This approach is robust even though some modifications are sometimes necessary to account for the mean stress effects or multi-axial loading [28-31] and when oxidation or creep interacts with fatigue [19,32,33]. A fatigue criterion inspired by the work of Constantinescu et al. [16] on the aluminium is used. This criterion is based on the inelastic dissipated energy density per cycle determined at stabilized hysteresis loops, $\Delta W_{\text {ine }}$ as following:

$\Delta W_{\text {ine }}=A N^{\beta}$
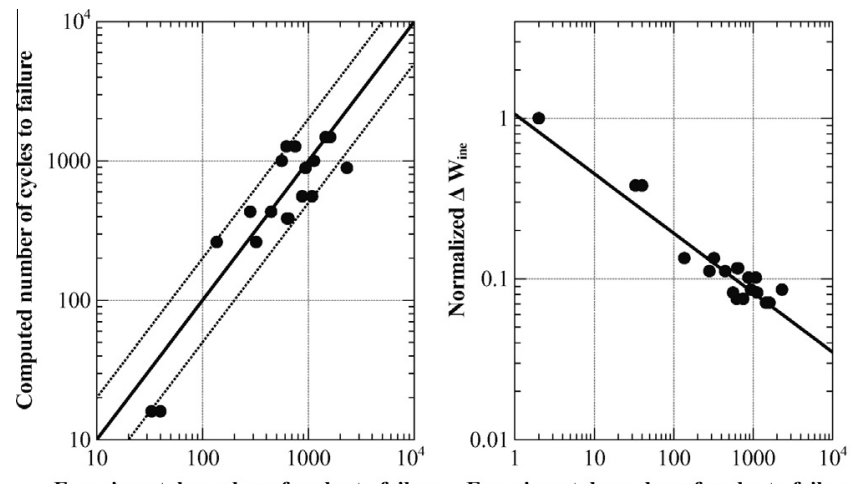

Experimental number of cycles to failure with

$\Delta W_{\text {ine }}=\int_{\text {cycle }} \underline{\underline{\sigma}}: \underline{\underline{\dot{\varepsilon}}} d t$

$A$ and $\beta$ are two material parameters. We estimate these parameters by using the dissipated energy obtained by the FEM simulation by Abaqus ${ }^{\circledR}$ of the pistons tested in these investigations applying the above mentioned constitutive model. This criterion is successful in prediction of the LCF test lifetime as seen in Fig. 6 even if the life prediction presents some scattering.

\section{Results and discussions}

\subsection{Thermal loading}

Fig. 7 shows a simulated thermal map by Abaqus ${ }^{\circledR}$ for a piston operating for an engine in a steady state condition, i.e. after several seconds of engine operations working at a maintained high speed. The maximum temperature for the piston is located at the edge of its bowl that achieves $374{ }^{\circ} \mathrm{C}$. A slight asymmetry is observed on the thermal field with higher temperatures at the recesses of the exhaust valves. Indeed, as the exhaust gases are hotter than intake ones, they tend to increase the local thermal loading of the structure. This asymmetry is neglected in the rest of the study. The spatial gradient is oriented along a direction perpendicular to the surface of the bowl and a drop of about $80^{\circ} \mathrm{C}$ is observed inward from the surface in a depth of $15 \mathrm{~mm}$. The cooling gallery also has a significant impact and creates a vertical gradient of the same

Fig. 6. Thermomechanical fatigue criterion precision. 

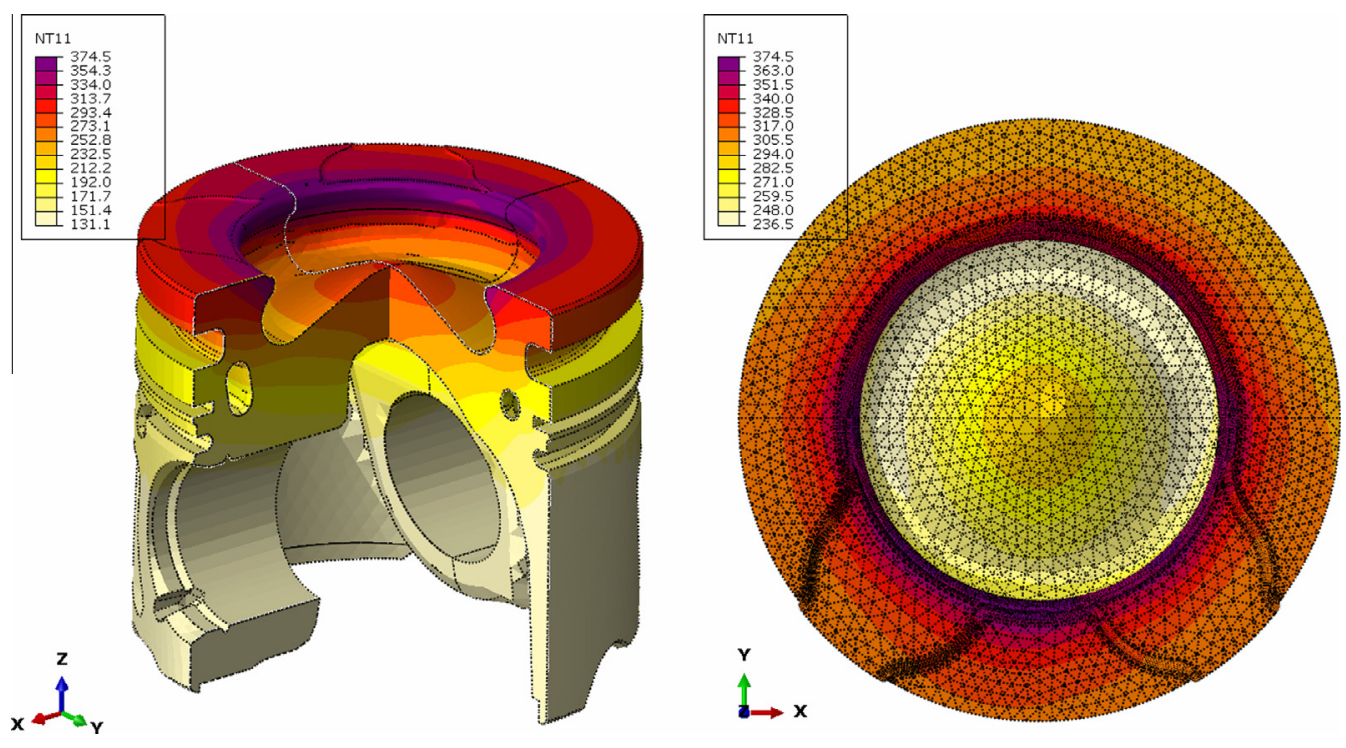

Fig. 7. Maximal temperature and gradients cartography for a piston in engine use (cut and fire deck view).

magnitude. The steady state is reached in the bowl edge zone after $5 \mathrm{~s}$ of thermomechanical loading.

In view of these results, the loading cycle to be imposed to the piston is determined. The loading must ideally leads to $375{ }^{\circ} \mathrm{C}$ on surface at the edge of the bowl in $5 \mathrm{~s}$. As the simulated operating conditions are very severe, the experimental objective was fixed to $90 \%$ of this value, i.e. $340{ }^{\circ} \mathrm{C}$. The cooling part of the loading cycle involves the stop of the heating system until the temperature of the piston reaches the same value as at the beginning of the cycle only thanks to the water forced convection. A thermocouple placed at $2 \mathrm{~mm}$ from the edge of the bowl, is then used as a reference for measuring this temperature and thus estimating the time required for the cooling sequence. The proposed loading cycle induces in bench conditions the heating and cooling times of respectively 5.1 and $4.15 \mathrm{~s}$ for the heating and cooling parts. This results in a local temperature variation at this reference thermocouple between $105^{\circ} \mathrm{C}$ and $270{ }^{\circ} \mathrm{C}$. The maximum measured temperature at the edge of the piston bowl is $350^{\circ} \mathrm{C}$ (itself influenced by the cooling channel design as its shape and diameter vary continuously all around the piston. Fig. 8a and b shows the temperature evolution measured by the reference thermocouple during the third thermal cycle and also the thermal gradient induced in the piston inwards from the surface measured by an Infrared camera when the temperature of the thermal cycle is maximum.

A transient analysis corresponding to the heating and cooling phases of an engine bench test is performed and repeated for 3 cycles until the thermal map get stabilized. As shown in Fig. 8c, experimental thermal evolution stabilizes after a dozen cycles and the 15th cycle is taken as the reference stabilized cycle for further comparisons. These Comparisons are essentially made for a stabilized state both for numerical and experimental results and for maximal and minimal temperatures of the thermal cycle and so for temperature rates of concern. In the bowl edge zone, the standard size of a mesh element is $0.3 \mathrm{~mm}$ and the precise localisation for thermocouples therefore corresponds exactly to a mesh node and the comparison is thus precise and relevant. Fig. 8 shows that temperature evolution is accurately described by the FEM simulation even if some approximations were made. For the reference thermocouple, the differences in temperature never exceed $5 \%$. The surface gradient at maximal temperature is very well reproduced by the FEM computation which implies reliable boundary conditions for the numerical lifetime evaluations.
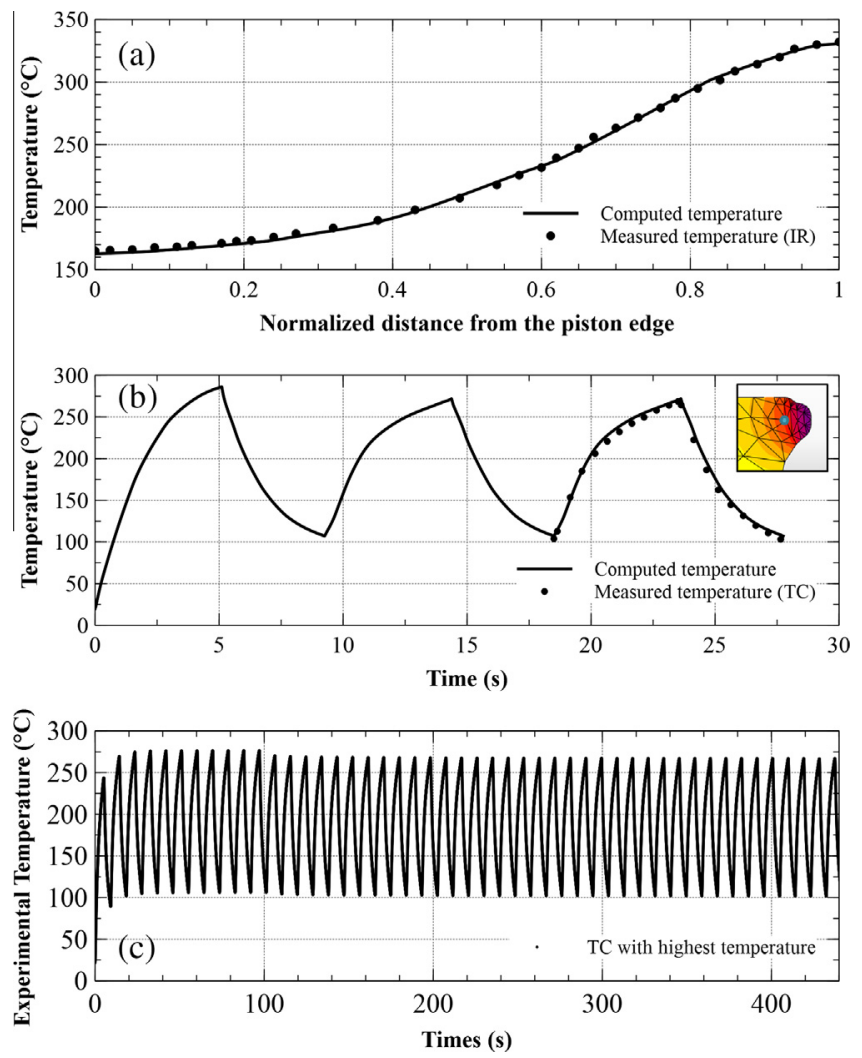

Fig. 8. Nodal temperature evolution in the reference thermocouple location Comparison between stabilized experimental and computed results.

Fig. 9 shows the detailed results of the thermal analysis for the maximum temperature. The computed gradients are circumferential with a maximum temperature at the edge of the bowl equal to $345^{\circ} \mathrm{C}$. The imposed thermal loading is slightly more severe in terms of thermal gradients than the engine loading but enables to reproduce on the structure the loading conditions that are derived from the engine environment. The vertical gradient imposed by the cooling channel is also present. The piston is then subjected to a temperature variation of the order of $150{ }^{\circ} \mathrm{C}$ over a 

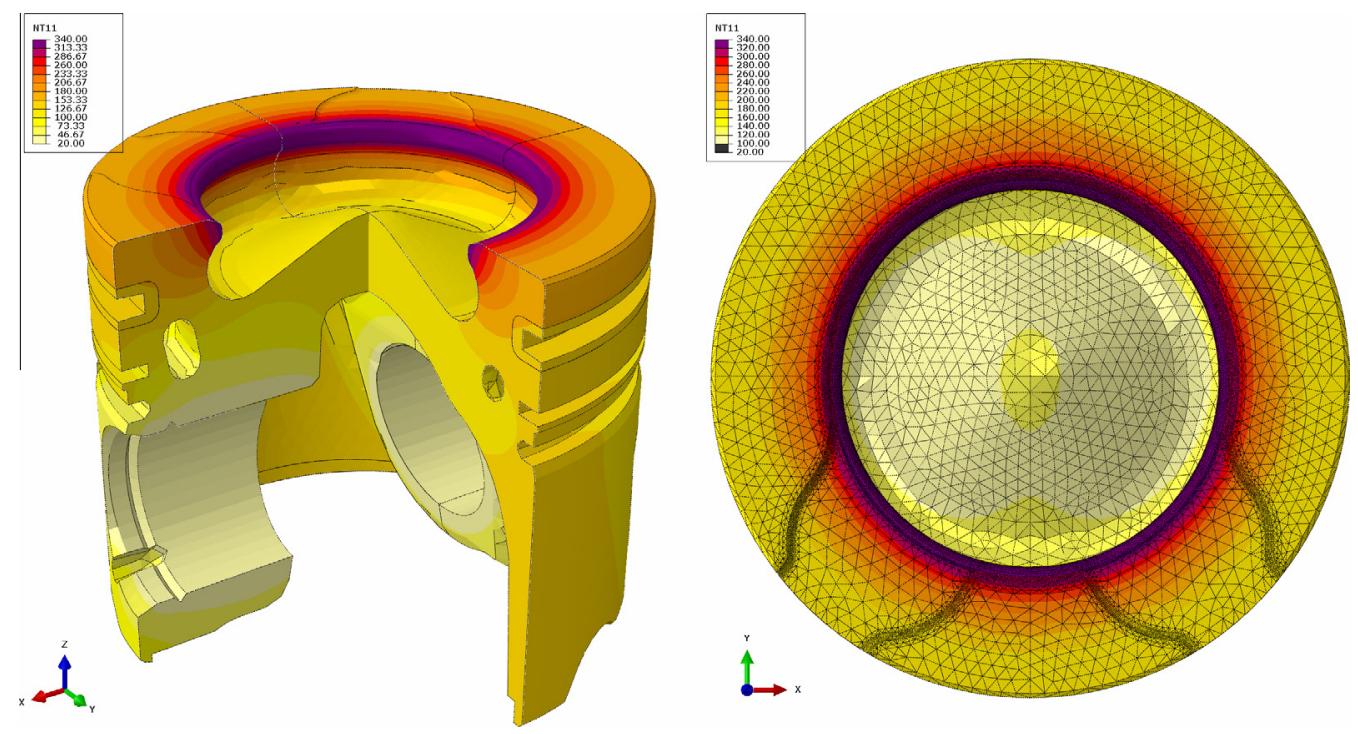

Fig. 9. Maximal temperature and gradients cartography for a piston in bench use (cut and fire deck view).

distance of $1 \mathrm{~cm}$, which concentrates the stress zones at the edge of the bowl and thus enables to more easily test geometry selections.

\subsection{Thermo-mechanical loading}

First a simple axisymmetric computation is undertaken to show that the milled zone has no influence in the fatigue development. Then a complete meshing 3D meshing and analysis are performed without supressing the milled zone. Fig. 10 presents calculated mechanical stress-strain hysteresis loops for an element of the bowl edge during the first 30 TF cycles. Upon TF cycling condition, the bowl edge undergoes an alternative compression/tension triaxial thermomechanical cyclic loading. The principal loading direction is the $y y$ one which is here examined. The loading starts with nil stress and strain at $20^{\circ} \mathrm{C}(\mathrm{A})$ and then undergoes a com-

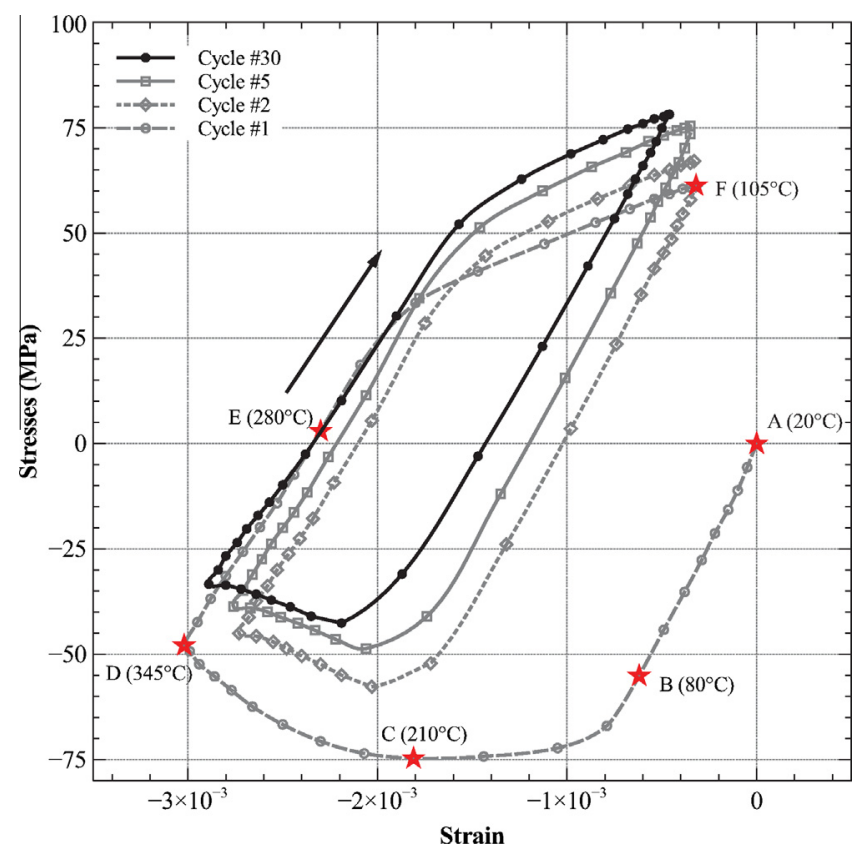

Fig. 10. Bench local loadings on the critical zone. pressive loading during heating-up according to the A-D path. The loading path is thermoelastic below $80^{\circ} \mathrm{C}(\mathrm{AB})$ and then is followed by a thermo-visco-plastic evolution up to $T_{\max }$ (BD). A compressive peak stress $(-75 \mathrm{MPa})$ is achieved at about $210^{\circ} \mathrm{C}(\mathrm{C})$ and beyond this temperature, the stress decreases while the mechanical strain continues to increase (CD). This behaviour is explained by the fall of the aluminium alloy mechanical properties beyond this temperature. During cooling period, stress increases toward tension when the behaviour of the alloy is governed mostly by a thermoelastic behaviour (DE). A visco-plastic yielding occurs again when the temperature decreases from $280^{\circ} \mathrm{C}$ to $105^{\circ} \mathrm{C}(\mathrm{EF})$. The maximal tensile stress is reached at $105^{\circ} \mathrm{C}$ at the end of the first TF cycle $(F)$ which, for the first loading cycle, does not coincide with the initial starting point (A). The plastic strain is less important in subsequent cycles that evolving to closed mechanical stress-strain loops meaning the mechanical state of the aluminium alloy evolves toward an asymptotic state (quasi steady state region) with a stress and strain behaviour due to visco-plastic shakedown phenomenon.

\subsection{Thermal fatigue damage}

Cracks initiation localisations are listed in Fig. 11. The first cracks start at areas the thermocouples are placed especially in the zone where 3 thermocouples (including the reference thermocouple) are positioned (for gradient measurement purpose). The reference sensor is indeed positioned $2 \mathrm{~mm}$ inward the edge of the bowl in an area where the thermal gradient is most drastic. After only 250 loading cycles, a crack appears and propagates rapidly in-depth to reach $9 \mathrm{~mm}$ where the crack propagation rate is very much reduced leading to the stabilisation of the crack progress. Four TF cracks appear in the valve recesses zones after $3520 \mathrm{TF}$ cycles. Other cracks appear later at 3550 cycles in another thermocouple localisation and subsequently at $4500 \mathrm{TF}$ cycles in a slightly less stressed zone. It must be emphasised that the cracks initiation and propagation in particular when the cracks are long tend to produce heterogeneous induction heating by altering the electromagnetic fields. Heating is therefore concentrated around the crack lips (long crack $>7 \mathrm{~mm}$ ) which may compromise the use of induction in TF testing. Nevertheless, the main objectives of the TF testing rig developed here are validated. The experimental set-up indeed enables to generate a local damage in areas with 

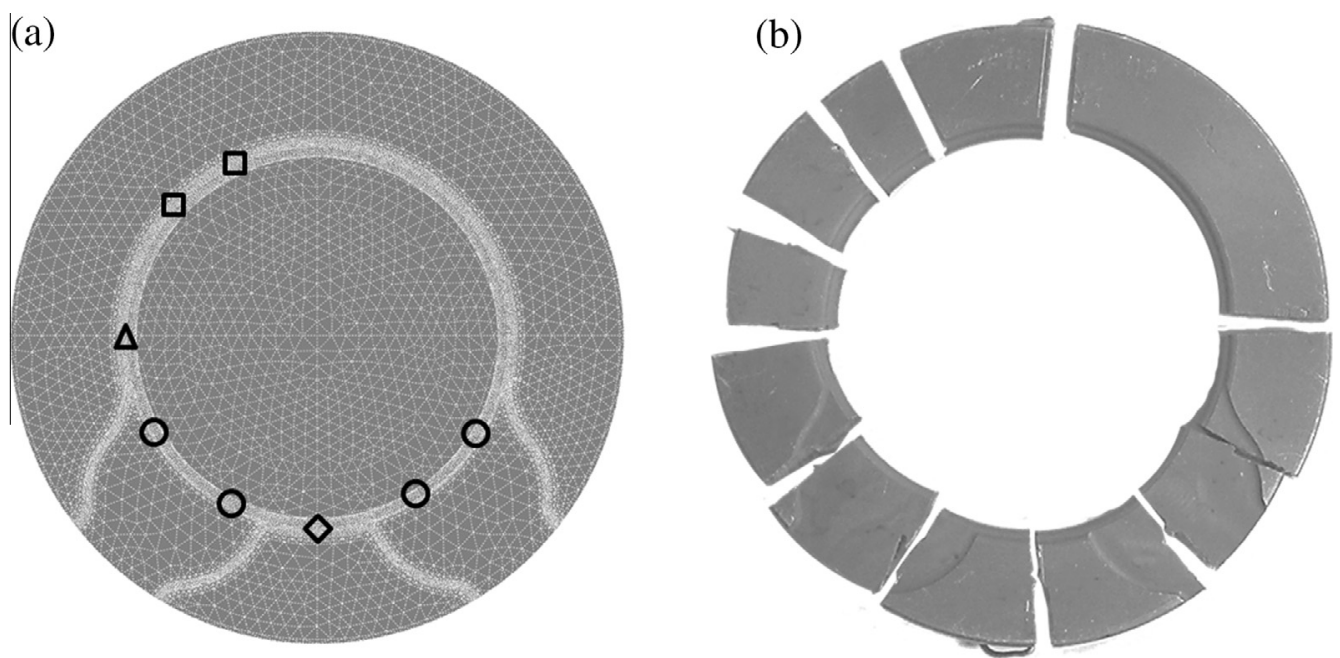

O Fatigue crack initiation at 3520 cycles

$\Delta$ Thermocouple crack initiation : early damage at 250 cycles : no influence on other crack initiation

$\diamond$ Thermocouple crack initiation at 3550 cycles

口 Fatigue crack initiation at 4500 cycles

Fig. 11. Crack sites provided by simulation (a) and experiment (b).
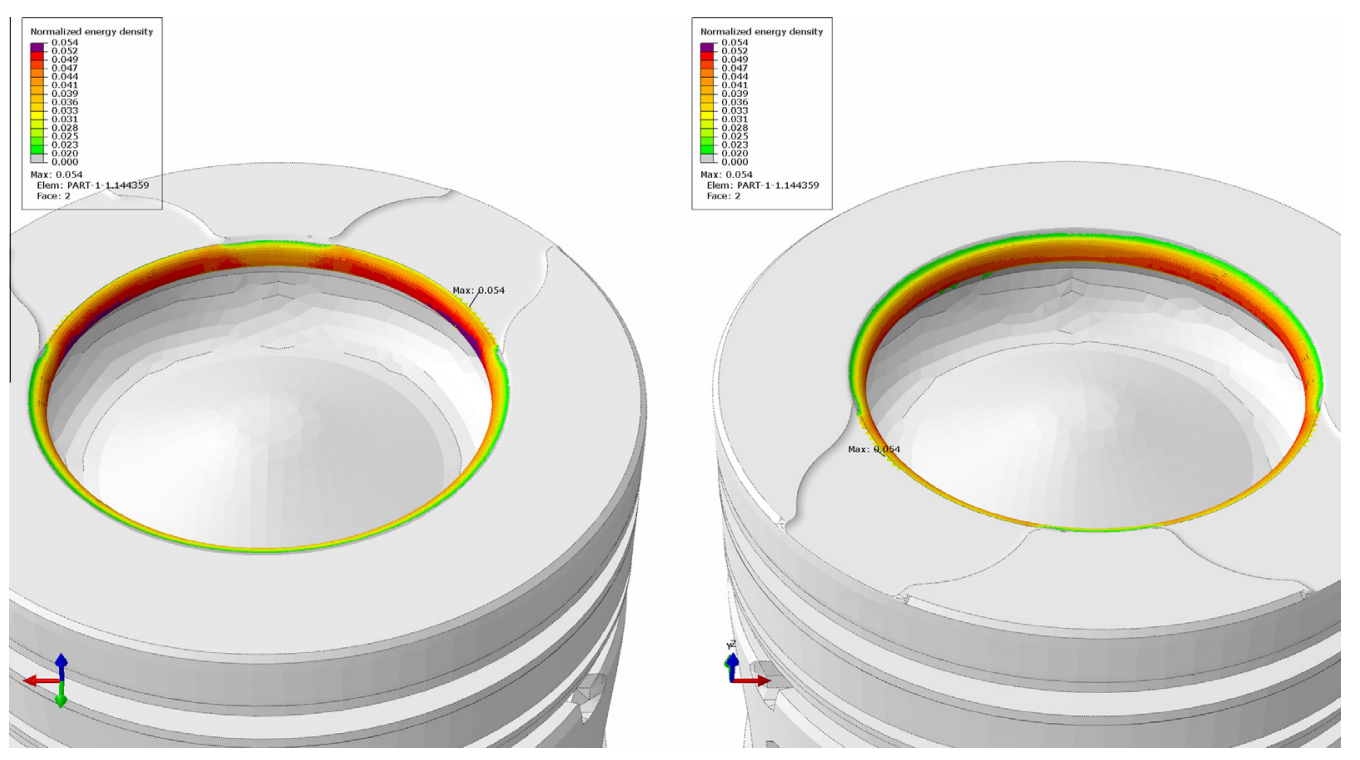

Fig. 12. Bench piston lifetime analysis. Crack initiation predicted zone.

severe thermal gradients and stress concentration. The thermomechanical fatigue damage criterion based on dissipated energy density per cycle is calculated using the last TF cycle that is considered as the stabilized cycle. The TF life curves are then expressed by the number of cycles to failure thanks to this criterion. Numerical estimation of these cracking zones is presented in Fig. 12.

The analyse of this figure shows a good correlation between simulation and experimental results. The FE numerical analysis using the EVP behaviour law and fatigue cyclic dissipated inelastic energy density criterion previously mentioned enables therefore to detect the most sensitive zones in term of resistance to TF cracking. Indeed, as it can be seen in Fig. 12, dissipated inelastic energy density reaches its maximal level in the valve recess areas which are, regardless the thermocouple holes (not meshed and not taken into account in the model), the first zones to be cracked experimentally. Moreover, the evolution of the dissipated energy density in the critical zone is completely stabilized at the 30th simulated TF cycle as seen in Fig. 10. It implies that the hypotheses of the fatigue design protocol are then verified and also there is thus a quite good agreement between modelling and experimental results. The proposed criterion leads indeed to an estimated lifetime of 3120 cycles, which is very close to the experimental value.

However, the presence of thermocouples disrupts the test analysis and thus extreme care must be taken to position them farther from the edge of the bowl and therefore from the influence of thermal gradients, in order to obtain doubtless results. Nevertheless, the proposed fatigue test is very complete and should, by working more in detail on the TF crack propagation and the role of the 
microstructure, facilitate the design of pistons against thermomechanical fatigue solicitations.

\section{Conclusions}

The proposed experimental set-up allows easy testing of the Thermal Fatigue resistance of a Diesel engine piston while the proposed numerical modelling helps to complete the experiments by detecting the most severe areas and their potential lifetime. The rig specifications (water cooling, simple heating by induction) are thus justified on several levels:

- The piston is an automotive part with small dimensions. Its overall size varies little and only the bowl undergoes strong geometrical variations while its width, height, axis sizes are subjected to few variability. The proposed bench is therefore suitable for all the kind of piston: only the coil should be adapted to the bowl diameter.

- The piston operating temperature is low enough to allow for different types of heating-up but induction conditions are chosen because they allow an axisymmetric and uniform heating of the edge of the bowl. Heating rates are also consistent with the actual use of the part (the constituent materials of the piston mainly aluminium alloys and steels - can see their temperature locally changing from 100 to $400{ }^{\circ} \mathrm{C}$ in seconds).

- The bench allows the use of pistons provided by commercial manufacturer and does not require the creation of specific specimens. Furthermore, materials solutions for pistons generally include the use of metal inserts or coatings as thermal barrier, which is closely connected to the geometry. In addition, the critical zone is an area where the microstructure and the fatigue resistance are strongly dependent on the casting and machining operation. The role of this particular microstructure is thus more easily analysed with specimens that are very close to the commercial part.

The TF rig presented here allows for many comparative studies and ranking of pistons and/or materials (with or without surface treatments) and gives a quick idea about the best compromise of design/process/material in terms of TF resistance. Such a TF protocol allows both exploring innovative material solutions, bowls geometry or different manufacturing processes and obtaining innovative piston design from tests easy to perform and representative of engine operations. One can also add that, as the heat loading is perfectly understood and numerically reproduced. Therefore the testing on bench can be a useful and relevant strategy to validate also the constitutive models and fatigue lifetime criteria. Finally, it should help to measure the scattering in terms of strength of thermo-mechanical fatigue for a given type of piston. Simple and inexpensive tests could therefore be carried out to estimate the production scattering and more easily quantify the risk of failure for customers as propose in [34].

\section{References}

[1] Ammar H, Samuel A, Samuel F. Effects of surface porosity on the fatigue strength of AE425 and PM390 hypereutectic Al-Si casting alloys at medium and elevated temperatures. Mater Sci Eng, A 2008;473(1-2):58-64.

[2] Martin GG. Failure of a stationary pump engine piston. J Fail Anal Prev 2004;4(1):37-9.

[3] Silva F. Fatigue on engine pistons-A compendium of case studies. Eng Fail Anal 2006;13(3):480-92.

[4] Ye H. An overview of the development of Al-Si-Alloy based material for engine applications. J Mater Eng Perform 2003;12(3):288-97.

[5] Okamoto H, Anno N, Itoh T. New computational and experimental stress analysis method for the design decision on optimum piston configuration of production engine, SAE Technical Paper 920065; 1992.
[6] Moffat AJ, Mellor BG, Chen CL, Thomson RC, Reed PAS. Microstructural analysis of fatigue initiation in Al-Si casting alloys. In: Poole WJ, Wells MA, Lloyd DJ, editors. Aluminium alloys 2006: innovation through research and technology. In: 10th international conference on aluminum alloys Switzerland, Trans Tech, 2006. p. 1083-8

[7] Yanxia W, Xinliang Q Kun H. The reliability analysis for the piston, connecting rod and crankshaft assembly of diesel engine. In: International conference on mechanical engineering and mechanics, Beijing, China; 2009.

[8] Hardy J, Lahjaily H, Besson M, Gastaldi P. Diesel combustion optimization at full load by combined CFD and single cylinder tests. SAE Technical Paper 200401-1402; 2004.

[9] Béard P, Mokaddem K, Baritaud T. Measurement and modeling of the flowfield in a DI diesel engine: effects of piston bowl shape and engine speed, SAE Technical Paper 982587; 1998.

[10] Halászi C, Gaier C, Dannbauer H. Fatigue life prediction of thermomachanically loaded engine components. In: 11th European automotive congress, Budapest; 2007.

[11] Gardyński L, Weroński A. Examination of diesel engine pistons cracking reasons. In: Proc. 10th international scientific conference "Communications on the edge of the milleniums", Silina; 1998.

[12] Song HW et al. Thermal fatigue on pistons induced by shaped high power laser. Part I: experimental study of transient temperature field and temperature oscillation. Int J Heat Mass Transf 2008;51(3-4):757-67.

[13] Song HW et al. Thermal fatigue on pistons induced by shaped high power laser. Part II: design of spatial intensity distribution via numerical simulation. Int J Heat Mass Transf 2008:51(3-4):768-78.

[14] Ebner R et al. Thermal fatigue behaviour of hot-work tool steels: heat checks nucleation and growth. Int J Microstruct Mater Prop 2008;3(2):182-94.

[15] Mielke S, Laslaz G, Gautier-Picard C. Aluminium alloy for component with high hot process mechanical strength. WO Patent WO/2006/056,686; 2006

[16] Constantinescu A, Charkaluk E, Lederer G, Verger L. A computational approach to thermomechanical fatigue. Int J Fatigue 2004;26(8):805-18.

[17] Szmytka F, Maitournam MH, Rémy L. An implicit integration procedure for an elasto-viscoplastic model and its application to thermomechanical fatigue design of automotive parts. Comput Struct 2013;119(1):155-65.

[18] Szmytka F, Rémy L, Maitournam H, Köster A, Bourgeois M. New flow rules in elasto-viscoplastic constitutive models for spheroidal graphite cast-iron. Int J Plast 2010;26(6):905-24.

[19] Rémy L. Thermal-mechanical fatigue (including thermal shock). In: Milne I, Ritchie RO, Karihaloo B, editors. Comprehensive structural integrity. Oxford: Pergamon; 2003. p. 113-99.

[20] Garud Y. A new approach to the evaluation of fatigue under multiaxial loadings. J Eng Mater Technol - Trans ASME 1981;103(2):118-25.

[21] Skelton RP. Energy criterion for high temperature low-cycle fatigue failure. Mater Sci Technol 1991;7(5):427-39.

[22] Tabibian S, Charkaluk E, Constantinescu A, Szmytka F, Oudin A. TMF-LCF life assessment of a lost foam casting A319 aluminum alloy. Int J Fatigue 2013;53(August):75-81.

[23] Tomkins B. Fatigue crack propagation - an analysis. Phil Mag 1968;18(155):1041-66

[24] Lamba H. U. of Illinois at Urbana-Champaign. Dept. of Theoretical, A. Mechanics, U. S. A. R. P. Agency, J-integral applied to fatigue crack initiation, T. \& A.M. report, Department of Theoretical and Applied Mechanics, University of Illinois; 1974.

[25] Chalant G, Remy L. The slip character and low cycle fatigue behaviour: the influence of F.C.C. twinning and strain-induced F.C.C. > H.C.P. martensitic transformation. Acta Metall 1980;28(1):75-88.

[26] Reuchet J, Rémy L. Fatigue oxidation in a superalloy - application to life prediction in high temperature low cycle fatigue. Metall Trans A - Phys Metall Mater Sci 1983;14(1):141-9.

[27] Rémy L, Alam A, Haddar N, Koester A, Marchal N. Growth of small cracks and prediction of lifetime in high-temperature alloys. In: Materials science and engineering a - structural materials properties microstructure and processing 468 (SI) McEvily symposium on fatigue and fracture of traditional and advanced materials, San Antonio, TX, MAR 12-16, 2006; 2007. p. 40-50.

[28] Maurel V, Rémy L, Dahmen F, Haddar N. An engineering model for low cycle fatigue life based on a partition of energy and micro-crack growth. Int J Fatigue 2009;31(5):952-61.

[29] Ostergren WJ. A damage function and associated failure equations for predicting hold time and frequency effects in elevated temperature, lowcycle fatigue. J Test Eval 1976;4:327-39.

[30] Amiable S, Chapuliot S, Constantinescu A, Fissolo A. A comparison of lifetime prediction methods for a thermal fatigue experiment. Int $\mathrm{J}$ Fatigue 2006;28(7):692-706.

[31] Park J, Nelson D. Evaluation of an energy-based approach and a critical plane approach for predicting constant amplitude multiaxial fatigue life. Int J Fatigue 2000;22(1):23-39.

[32] Rézaï-Aria F, Dambrine B, Rémy L. Thermal fatigue of MAR-M509 superalloyr. Part 2: evaluation of life prediction models. Fatigue Fract Eng Mater Struct $1988 ; 11(4): 291-302$

[33] Rézaï-Aria F, Rémy L. An oxidation fatigue interaction damage model for thermal fatigue crack growth. Eng Fract Mech 1989;34:283-94.

[34] Szmytka F, Oudin A. A reliability analysis method in thermomechanical fatigue design. Int J Fatigue 2013;53(August):82-91. 